\title{
CFTR channel opening by ATP-driven tight dimerization of its nucleotide-binding domains
}

\author{
Paola Vergani $^{1}$, Steve W. Lockless ${ }^{2}$, Angus C. Nairn ${ }^{3,4}$, and David C. Gadsby ${ }^{1}$ \\ ${ }^{1}$ Laboratory of Cardiac/Membrane Physiology, The Rockefeller University, New York, New York \\ 10021, USA \\ ${ }^{2}$ Laboratory of Molecular Neurobiology and Biophysics, The Rockefeller University, New York, New \\ York 10021, USA \\ ${ }^{3}$ Laboratory of Molecular and Cellular Neuroscience, The Rockefeller University, New York, New \\ York 10021, USA \\ ${ }^{4}$ Department of Psychiatry, Yale University, New Haven, Connecticut 06519, USA
}

\begin{abstract}
ABC (ATP-binding cassette) proteins constitute a large family of membrane proteins that actively transport a broad range of substrates. Cystic fibrosis transmembrane conductance regulator (CFTR), the protein dysfunctional in cystic fibrosis, is unique among $\mathrm{ABC}$ proteins in that its transmembrane domains comprise an ion channel. Opening and closing of the pore have been linked to ATP binding and hydrolysis at CFTR's two nucleotide-binding domains, NBD1 and NBD2 (see, for example, refs 1,2 ). Isolated NBDs of prokaryotic ABC proteins dimerize upon binding ATP, and hydrolysis of the ATP causes dimer dissociation ${ }^{3-5}$. Here, using single-channel recording methods on intact CFTR molecules, we directly follow opening and closing of the channel gates, and relate these occurrences to ATP-mediated events in the NBDs. We find that energetic coupling ${ }^{6}$ between two CFTR residues, expected to lie on opposite sides of its predicted NBD1-NBD2 dimer interface, changes in concert with channel gating status. The two monitored side chains are independent of each other in closed channels but become coupled as the channels open. The results directly link ATP-driven tight dimerization of CFTR's cytoplasmic nucleotide-binding domains to opening of the ion channel in the transmembrane domains. This establishes a molecular mechanism, involving dynamic restructuring of the NBD dimer interface, that is probably common to all members of the $\mathrm{ABC}$ protein superfamily.
\end{abstract}

Crystal structures of most ABC-protein NBDs determined so far share the same fold ${ }^{7,8}$ with a core subdomain ('head') that binds the ATP, and an $\alpha$-helical subdomain ('tail') that includes the ABC-specific signature sequence (LSGGQ). Dimeric structures revealed nucleotide-bound NBD homodimers in rotationally symmetric 'head-to-tail' arrangement, enclosing two ATP molecules within interfacial composite sites, each comprising conserved ATP-binding motifs from the head of one monomer and signature sequence residues from the tail of the other ${ }^{3,5}$,

9,10 . On the basis of this structural evidence and biochemical studies of reversible dimerization of isolated NBDs ${ }^{4,5,11-13}$, opening and closing of CFTR channels can be interpreted ${ }^{14}$ in terms

(C) 2005 Nature Publishing Group

Correspondence and requests for materials should be addressed to D.C.G. (gadsby@ @rockefeller.edu) or to P.V. (paola.vergani@ rockefeller.edu).

Supplementary Information accompanies the paper on www.nature.com/nature.

Competing interests statement The authors declare that they have no competing financial interests. 
of cycles of NBD1-NBD2 dimerization and dissociation, induced by ATP binding and hydrolysis, respectively (Fig. 1a). Opening of a phosphorylated CFTR $\mathrm{Cl}^{-}$channel seems to require ATP binding to both composite sites because, at low [ATP], mutations expected to weaken ATP binding can make nucleotide occupancy at either site rate-limiting for channel opening ${ }^{14}$. In addition, interfering with hydrolysis prevents the normal rapid closing of CFTR channels ${ }^{1,2,14}$. Because photolabelling studies show that ATP can remain at the NBD1-head site for several minutes without being hydrolysed ${ }^{15,16}$, whereas a CFTR-channel gating cycle lasts only seconds, channel opening and closing seem to be timed by nucleotide binding and hydrolysis at the composite site incorporating the NBD2 head, which we call the NBD2 catalytic site ${ }^{14,15}$.

Evidence supporting our speculation (Fig. 1a) that the CFTR-channel open state corresponds to the dimerized NBD conformation is provided by the approximately 1,000-fold stabilization of the open-burst state that results from mutation of the possible catalytic base ${ }^{4,17}$, Glu 1371 (a glutamate in the NBD2-head 'Walker B' motif), to Gln (E1371Q; Fig. 1b). Because CFTR channels do not open without ATP (see below), current decay upon the removal of ATP reflects channel closing, and its time course measures the open burst duration; closing was complete within about $1 \mathrm{~s}$ of ATP withdrawal for wild-type (WT) CFTR channels (mean open burst duration was less than $0.5 \mathrm{~s}$ ), but had a time constant of $411 \pm 64 \mathrm{~s}$ (mean \pm s.e.m., $n=16$ ) for mutant E1371Q channels (Fig. 1b). Correspondingly, in isolated NBD subunits impairment of ATP hydrolysis by the homologous Glu-to-Gln mutation induces the formation of very stable ATP-bound homodimers ${ }^{4,5,11,12}$.

To test the dynamic domain rearrangements underlying the model (Fig. 1a), we investigated the interaction between residues on opposite sides of the predicted NBD1-NBD2 heterodimer interface. In crystals of ATP-bound prokaryotic NBD homodimers (MJ0796, the

hyperthermophylic archaeon homologue of LolD, part of a putative lipoprotein transporter ${ }^{5}$, and MalK, the NBD of the maltose transporter of Escherichia coli ${ }^{10}$ ) a hydrogen bond connects the residues corresponding to CFTR's Arg 555 (three positions after the signature sequence: LSGGQRAR) in the NBD1 tail, and Thr 1246 (within the 'Walker A' phosphate-binding loop, GRTGSGKS) in the NBD2 head. We chose to target this pair of residues on the basis of a statistical analysis (Fig. 2b) of more than 10,000 NBD sequences, which suggests that positions corresponding to CFTR's Arg 555 and Thr 1246 are functionally coupled ${ }^{18}$. Partitioning the total multiple sequence alignment into subsets, on the basis of the side chain present at the site corresponding to Arg 555, yields one major subset with arginine, and another with lysine, at that position, both of which are potential hydrogen bond donors. The distribution of potential acceptor side chains at the position corresponding to Thr 1246 differs in these two subsets, the shorter lysine donor being more frequently paired with the longer asparagine acceptor, and the longer arginine donor being more frequently paired with the shorter serine acceptor (or the equivalent threonine, as found in CFTR) (Fig. 2b). This striking covariance suggests that the two sites have been subject to evolutionary pressure as a pair, rather than individually, so as to retain the ability to form a hydrogen bond that precisely distances the two $\alpha$-carbons (Fig. 2a) in the dimer structure.

We first mutated Arg 555, and found that the charge-removing mutation R555Q slowed channel closing (mean open burst duration was $3.20 \pm 0.35 \mathrm{~s}(n=18)$ for R555Q, compared with 0.43 $\pm 0.02 \mathrm{~s}(n=32)$ for WT; Fig. $2 \mathrm{c})$, consistent with Arg 555 being part of the composite NBD2 catalytic site, where the ATP hydrolysis that times closing of WT channels occurs. Possibly, the positive charge of Arg 555 normally helps to stabilize the partial negative charges developed on the $\beta$ - and/or $\gamma$-phosphate in the transition state for that ATP hydrolysis ${ }^{19}$; an equivalent mutation impairs ATP hydrolysis in the ABC multidrug transporter P-glycoprotein ${ }^{20}$.

Accordingly, the charge-conserving mutation R555K did not affect open burst duration (mean $0.39 \pm 0.04 \mathrm{~s}(n=26))$. However, it substantially prolonged closed interburst duration (inversely 
related to opening rate) from $2.29 \pm 0.46 \mathrm{~s}(n=16)$ for WT to $8.53 \pm 1.23 \mathrm{~s}(n=15)$ for R555K (Fig. 2c, all measured at saturating [ATP]). On the basis of the evolutionary evidence suggesting the involvement of the side chain at this position in a conserved interaction (Fig. $2 \mathrm{~b}$ ), this slowing of channel opening could be explained if the R555K mutation were to weaken or remove a hydrogen bond between NBD1 and NBD2 that is absent in the closed, ground, state but present in the transition state for the channel opening reaction; the resulting destabilization of the transition state would increase the activation free energy $\left(\Delta G^{\ddagger}\right)$ for channel opening and hence decrease the opening rate.

To quantify this suspected interaction between Arg 555 and Thr 1246 side chains (Fig. 2a), we applied double mutant-cycle analysis ${ }^{6,21}$ (see Supplementary Information), after mutating Arg 555 to Lys and Thr 1246 to Asn, both individually and jointly. The WT protein, the two single mutants and the double mutant form the corners of a thermodynamic cycle (Fig 3a and Fig 4a, d). If the two residues do not interact, the effects of mutating Arg 555 to Lys should be the same in a Thr 1246 background as in a T1246N background (and vice versa); that is, the effects of the single mutations should be independent and hence additive, and mutation-linked changes on parallel sides of the cycles should thus be equal. Any difference signifies, and quantifies, energetic coupling $\left(\Delta \Delta G_{\text {int }}\right)$ between the two residues. Because changes in any pathindependent variable can be used to evaluate the effects of the mutations, we used different kinetic measurements to assess energetic coupling between residues at positions 555 and 1246 at different stages of the channel gating cycle.

We first examined coupling in closed channels. The opening rate of WT CFTR shows simple Michaelis-Menten dependence on [ATP] (Fig. 3c), most probably reflecting ATP interaction with the NBD2-head binding site ${ }^{15}$. The measured maximal opening rate is relatively slow $\left(0.27 \mathrm{~s}^{-1}\right.$, for pre-phosphorylated channels ${ }^{14}$; step $\mathrm{C}_{2}$ to $\mathrm{O}$ in Fig. 1a), allowing the preceding ATP binding step $\left(\mathrm{C}_{1}\right.$ to $\left.\mathrm{C}_{2}\right)$ to reach a steady state not far from equilibrium. Consequently, the apparent affinity for ATP $\left(K_{0.5}\right)$ provides a good estimate of its dissociation constant at the NBD2 site on the closed channel $\left(\mathrm{C}_{2}\right)$. Changes in $K_{0.5}$ could therefore be used to quantify the effects of mutations and so assess coupling between Arg 555 and Thr 1246 in closed channels (Fig. 3). The apparent affinity for ATP was little influenced by the mutation R555K (R555K $K_{0.5}=71 \pm 14 \mu \mathrm{M}$ versus WT $K_{0.5}=55 \pm 5 \mu \mathrm{M}$ ), but was reduced by the mutation T1246N (T1246N $K_{0.5}=261 \pm 49 \mu \mathrm{M}$ ) by the same extent in the WT background as in the R555K background (R555K T1246N $K_{0.5}=257 \pm 51 \mu \mathrm{M}$ ). The closely similar effects of mutations on parallel sides of the double mutant cycle signify a coupling energy not significantly different from zero $\left(\Delta \Delta G_{\text {int(unbound-bound) }}=0.31 \pm 0.55 \mathrm{kT}\right)$. So, either Arg 555 and Thr 1246 do not interact when CFTR channels are closed, or they do interact but with identical coupling energies before, and after, binding of the second ATP (that is in state $\mathrm{C}_{1}$ and in state $\mathrm{C}_{2}$ ). But, given that the residue corresponding to Thr 1246 is observed to directly contact the $\gamma$-phosphate oxygens in all ATP-bound crystal structures, both monomeric and dimeric ${ }^{3,5,10,22,23}$, it is highly improbable that there is significant energetic coupling between Arg 555 and Thr 1246 that is unaffected by ATP binding. We therefore conclude that the two target side chains do not interact in the closed-channel conformations (either with or without bound ATP), and hence that ATP binding occurs before the formation of a closely apposed NBD1-NBD2 dimer. Indeed, in dimeric crystals the bound ATP molecules are buried within the interface ${ }^{5,10}$, implying that access to the binding sites must occur in a different conformation.

We next tested for energetic coupling as CFTR channels approach the open burst state, by determining changes in activation free energies for channel opening (in the presence of saturating [ATP]). The slowing of opening caused by the R555K mutation (Fig. 2c, above) corresponded to a $1.4 \pm 0.4 k T$ increase in the activation energy barrier. The T1246N mutation also greatly slowed channel opening, increasing the energy barrier by $2.5 \pm 0.4 k T$. However, fast opening was partly restored when the two mutations were introduced simultaneously (Fig. 
$4 \mathrm{~b}, \mathrm{c})$. Comparing changes in activation-energy barrier height caused by each mutation in the WT, and in the mutant background, we obtain an energetic coupling between residues at positions 555 and 1246 of $\Delta \Delta G_{\text {int(opening) }}^{\ddagger}$ of $-2.7 \pm 0.5 k T$. The negative sign is consistent with Arg 555 and Thr 1246 in WT CFTR forming a stabilizing interaction, present in the transition state but not in the closed, ground, state (the above conclusion that these two residues do not interact in closed channels allows us to rule out the alternative interpretation, that the negative coupling energy could arise from a destabilizing interaction in the closed state that is lost in the transition state for channel opening; see also Supplementary Information). The presence of the postulated hydrogen bond between Arg 555 and Thr 1246 in the transition state could represent that stabilizing interaction: either mutation alone would remove the hydrogen bond and destabilize the transition state, whereas both mutations together would restore favourable geometry for hydrogen bond formation (Fig. 2a) and so speed up channel opening.

Interaction between Arg 555 and Thr 1246 in the open burst state, rather than approaching it, was monitored by measuring channel open probability $\left(P_{\mathrm{o}}\right)$ to estimate the relative stability of the closed and open states, after reducing the channel gating scheme to a simple closed-open equilibrium. For the scheme in Fig. 1a, the latter condition holds, at saturating [ATP], if channel closing from open bursts by means of the hydrolytic pathway $\left(\mathrm{O}\right.$ to $\left.\mathrm{C}_{1}\right)$ is precluded. In other ABC-ATPases, mutating the key lysine in the phosphate-binding loop to arginine drastically reduces or abolishes hydrolysis (see, for example, ref. ${ }^{24}$ ) and, as would be predicted if hydrolysis at CFTR's NBD2 catalytic site were markedly slowed, CFTR channels carrying the corresponding mutation (K1250R) have prolonged open burst durations (Fig. 4e; mean time constant of current decay upon ATP removal $\tau=9.3 \pm 0.5 \mathrm{~s} ; n=49$ ). Introducing the T1246N mutation into the K1250R background decreased $P_{\mathrm{o}}$, corresponding to destabilization of the open burst state by $2.5 \pm 1.0 \mathrm{kT}$ with respect to the closed state. However, adding the R555K mutation to T1246N-K1250R channels restored high stability of the open state (Fig. 4e, f). The coupling energy obtained from the mutant cycle (Fig. 4d) was again negative in sign $\left(\Delta \Delta G_{\text {int }}\right.$ (open-closed) $\left.=-2.4 \pm 1.0 k T\right)$, which is consistent with the presence of a stabilizing interaction (for example a hydrogen bond) between Arg 555 and Thr 1246 in the open burst state that is absent from the closed state.

Thus, as conformational changes gate CFTR's transmembrane $\mathrm{Cl}^{-}$-ion permeation pathway from closed to open, two residues, on opposite sides of the anticipated NBD1-NBD2 heterodimer interface, go from being independent to being energetically coupled, most probably by forming a hydrogen bond. The changes in the relative positions of CFTR's NBD1 and NBD2 implied by our results need not be large. They need be no larger than the relatively small differences observed between members of NBD pairs, juxtaposed in a 'head-to-tail' fashion, in crystal structures of nucleotide-bound versus nucleotide-free conformations. The residues corresponding to Arg 555 and Thr 1246 are close enough (2.7 $\AA$ ) to form a hydrogen bond in the ATP-bound crystals of LolD (MJ0796; ref. ${ }^{5}$ ) and MalK (PDB ID no. 1Q12; ref. ${ }^{10}$ ) and are found only a few ångströms further apart in nucleotide-free structures (for example, 5.7 ̊ in BtuCD (ref. ${ }^{9}$ ); 8.5 ̊ in MalK, PDB ID no. 1Q1B (ref. ${ }^{10}$ )), in which the NBDs share a limited contact surface but are kept in a dimeric arrangement by interactions occurring through contiguous domains (see also ref. ${ }^{13}$ ). Nevertheless, however small the local change at CFTR's NBD2 catalytic site, it is large enough to cause transmission of the long-range signal that results in opening of the channel pore.

By clearly linking NBD dimerization state to the disposition of the transmembrane domains in an intact, functioning, human $\mathrm{ABC}$ protein, our results establish dynamic NBD dimerization as the molecular mechanism that couples ATP binding and hydrolysis cycles to cyclic changes in the transmembrane domains. Conservation of the structural underpinnings of the hydrogen bond highlighted here implies that NBD dimerization occurs during the duty cycle of most, if 
not all, $\mathrm{ABC}$ proteins, although with subfamily-specific consequences. Thus, the tightly dimerized NBD conformation corresponds to the open-burst state in CFTR channels (Fig. 1), but in exporters or importers to conformations of the membrane domains that release drugs to the exterior ${ }^{25}$, or receive substrate from the substrate-binding protein ${ }^{26}$, respectively, and in DNA-repair ABC ATPases to relevant conformations in the DNA-binding regions ${ }^{27}$.

\section{Methods}

\section{Oocyte expression system and experimental set-up}

Human epithelial CFTR was mutated in an oocyte expression vector (pGEMHE-WT ${ }^{14}$ ) using QuikChange (Stratagene), and DNA templates were linearized with NheI before RNA transcription in vitro (mMessage mMachine; Ambion). Inside-out patches were excised from Xenopus laevis oocytes expressing WT or mutant CFTR channels and currents were recorded as described ${ }^{14}$. Bath (cytosolic) solution contained (in mM): $138 \mathrm{~N}$-methyl-d-glucamine (NMDG), $2 \mathrm{MgCl}_{2}, 5$ HEPES, 0.5 EGTA, 136 sulphamic acid (pH 7.1). Pipette (extracellular) solution contained (in mM): $138 \mathrm{NMDG}, 2 \mathrm{MgCl}_{2}, 5 \mathrm{HEPES}, 136 \mathrm{HCl}$ (pH 7.4). Membrane potential was held at $\pm 50 \mathrm{mV}$ ( $-50 \mathrm{mV}$ pipette potential), and outward currents were filtered at $200 \mathrm{~Hz}$ and digitized at $1 \mathrm{kHz}$. Bath solution flowed continuously (about $0.5 \mathrm{ml} \mathrm{min}^{-1}$ ) and solution was exchanged by using computer-driven solenoid valves. Channels were activated by exposure to $5 \mathrm{mM}$ ATP, magnesium salt, in the presence of $300 \mathrm{nM}$ purified cAMPdependent protein kinase catalytic subunit.

\section{Data analysis}

Digitized records were baseline-subtracted and idealized by half-amplitude threshold crossing; the events list was used to extract mean open burst $\left(\tau_{\mathrm{b}}\right)$ and closed interburst $\left(\tau_{\mathrm{ib}}\right)$ dwell times by simultaneous maximum-likelihood fitting to dwell-time histograms at all conductance levels ${ }^{28}$. Openings of CFTR channels are 'open bursts' occasionally interrupted by short-lived (non-conducting) 'flickery' closures, with low frequency and brief duration independent of ATP binding and hydrolysis. We refer to the 'open burst' as a single, composite state: channels 'open' upon entering a burst and 'close' upon entering the long-lived, interburst, closed state. However, the maximum-likelihood fit analysis yields estimates for all parameters, including flicker duration $\left(\tau_{\mathrm{F}}\right)$ and number of flickers per burst $\left(n_{\mathrm{F}}\right)$ (see Supplementary Table).

An artificial dead time of $4.5 \mathrm{~ms}$ was imposed to implement a correction for events missed because of limited bandwidth ${ }^{28}$. To obtain $\tau_{\text {ib }}$ measurements, only patches containing at most two simultaneously open channels were used. For constructs with very low $P_{\mathrm{o}}(\mathrm{R} 555 \mathrm{~K}$ and T1246N), we could not exclude the presence of unseen channels in the patch (even though the records lasted on average $6-7 \mathrm{~min}$ ). The prolonged $\tau_{\mathrm{ib}}$ values we extract for R555K and T1246N channels are therefore most probably underestimates, and the real effects of the mutations are more severe (and, hence, $\left|\Delta \Delta G_{\text {int(opening) }}^{\ddagger}\right|$ is actually larger) than the values we report.

$P_{\mathrm{o}}$ values (Fig. 4e, f) were estimated, on the assumption that channels are identical and independent and that the number of open channels in the patch is a random variable with binomial distribution, from the relationship between mean current $\left(I=N P_{\mathrm{o}} i\right.$, where $I$ is the mean current, $N$ is the number of channels present in the patch, and $i$ is the single-channel current) and variance of the current $\left(\sigma^{2}(I)=N i^{2} P_{\mathrm{o}}\left(1-P_{\mathrm{o}}\right)\right.$, where $\sigma^{2}(I)$ is the variance of the current).

Relative opening rates (Fig. 3c) were obtained as described ${ }^{14}$. In brief, the maximum-likelihood fit was performed on the assumption that the number of channels present in a given patch $(N)$ was equivalent to the maximum number of simultaneously open channels observed, during all test and reference conditions, in that patch. The opening rate obtained from the segments during 
exposure to the test [ATP] was then normalized to that obtained during the bracketing exposures to 5mM ATP, giving a relative value that was little sensitive to errors in our estimate of $N$.

\section{Supplementary Material}

Refer to Web version on PubMed Central for supplementary material.

\section{Acknowledgements}

We thank L. Csanády and G. Szakács for discussion. The work was supported by an NIH grant to D.C.G.

\section{References}

1. Gunderson KL, Kopito RR. Conformational states of CFTR associated with channel gating: the role of ATP binding and hydrolysis. Cell 1995;82:231-239. [PubMed: 7543023]

2. Carson MR, Travis SM, Welsh MJ. The two nucleotide-binding domains of cystic fibrosis transmembrane conductance regulator (CFTR) have distinct functions in controlling channel activity. J. Biol. Chem 1995;270:1711-1717. [PubMed: 7530246]

3. Hopfner KP, et al. Structural biology of Rad50 ATPase: ATP-driven conformational control in DNA double-strand break repair and the ABC-ATPase superfamily. Cell 2000;101:789-800. [PubMed: 10892749]

4. Moody JE, Millen L, Binns D, Hunt JF, Thomas PJ. Cooperative, ATP-dependent association of the nucleotide binding cassettes during the catalytic cycle of ATP-binding cassette transporters. J. Biol. Chem 2002;277:21111-21114. [PubMed: 11964392]

5. Smith PC, et al. ATP binding to the motor domain from an ABC transporter drives formation of a nucleotide sandwich dimer. Mol. Cell 2002;10:139-149. [PubMed: 12150914]

6. Serrano L, Horovitz A, Avron B, Bycroft M, Fersht AR. Estimating the contribution of engineered surface electrostatic interactions to protein stability by using double-mutant cycles. Biochemistry 1990;29:9343-9352. [PubMed: 2248951]

7. Davidson AL, Chen J. ATP-binding cassette transporters in bacteria. Annu. Rev. Biochem 2004;73:241-268. [PubMed: 15189142]

8. Higgins CF, Linton KJ. The ATP switch model for ABC transporters. Nature Struct. Mol. Biol 2004;11:918-926. [PubMed: 15452563]

9. Locher KP, Lee AT, Rees DC. The E. coli BtuCD structure: a framework for ABC transporter architecture and mechanism. Science 2002;296:1091-1098. [PubMed: 12004122]

10. Chen J, Lu G, Lin J, Davidson AL, Quiocho FA. A tweezers-like motion of the ATP-binding cassette dimer in an ABC transport cycle. Mol. Cell 2003;12:651-661. [PubMed: 14527411]

11. Janas E, et al. The ATP hydrolysis cycle of the nucleotide-binding domain of the mitochondrial ATPbinding cassette transporter Mdl1p. J. Biol. Chem 2003;278:26862-26869. [PubMed: 12746444]

12. Verdon G, et al. Formation of the productive ATP- $\mathrm{Mg}^{2+}$-bound dimer of GlcV, an ABC-ATPase from Sulfolobus solfataricus. J. Mol. Biol 2003;334:255-267. [PubMed: 14607117]

13. Horn C, Bremer E, Schmitt L. Nucleotide dependent monomer/dimer equilibrium of OpuAA, the nucleotide-binding protein of the osmotically regulated ABC transporter OpuA from Bacillus subtilis. J. Mol. Biol 2003;334:403-419. [PubMed: 14623183]

14. Vergani P, Nairn AC, Gadsby DC. On the mechanism of MgATP-dependent gating of $\mathrm{CFTRCl}^{-}$ channels. J. Gen. Physiol 2003;121:17-36. [PubMed: 12508051]

15. Basso C, Vergani P, Nairn AC, Gadsby DC. Prolonged nonhydrolytic interaction of nucleotide with CFTR's $\mathrm{NH}_{2}$-terminal nucleotide binding domain and its role in channel gating. J. Gen. Physiol 2003;122:333-348. [PubMed: 12939393]

16. Aleksandrov L, Aleksandrov AA, Chang XB, Riordan JR. The first nucleotide binding domain of cystic fibrosis transmembrane conductance regulator is a site of stable nucleotide interaction, whereas the second is a site of rapid turnover. J. Biol. Chem 2002;277:15419-15425. [PubMed: 11861646] 
17. Tombline G, Bartholomew LA, Urbatsch IL, Senior AE. Combined mutation of catalytic glutamate residues in the two nucleotide binding domains of P-glycoprotein generates a conformation that binds ATP and ADP tightly. J. Biol. Chem 2004;297:31212-31220. [PubMed: 15159388]

18. Lockless SW, Ranganathan R. Evolutionarily conserved pathways of energetic connectivity in protein families. Science 1999;286:295-299. [PubMed: 10514373]

19. Knowles J. Enzyme-catalyzed phosphoryl transfer reactions. Annu. Rev. Biochem 1980;49:877-919. [PubMed: 6250450]

20. Bakos É, et al. Characterization of the human multidrug resistance protein containing mutations in the ATP-binding cassette signature region. Biochem. J 1997;323:777-783. [PubMed: 9169612]

21. Fersht, A. Structure and Mechanism in Protein Science. New York: W. H. Freeman; 1999.

22. Hung LW, et al. Crystal structure of the ATP-binding subunit of an ABC transporter. Nature 1998;396:703-707. [PubMed: 9872322]

23. Verdon G, Albers SV, Dijkstra BW, Driessen AJM, Thunnissen A-MW. Crystal structures of the ATPase subunit of the glucose ABC transporter from Sulfolobus solfataricus: nucleotide-free and nucleotide-bound conformations. J. Mol. Biol 2003;330:343-358. [PubMed: 12823973]

24. Lerner-Marmarosh N, Gimi K, Urbatsch IL, Gros P, Senior AE. Large scale purification of detergentsoluble P-glycoprotein from Pichia pastoris cells and characterization of nucleotide binding properties of wild-type, Walker A, and Walker B mutant proteins. J. Biol. Chem 1999;274:3471134718. [PubMed: 10574938]

25. Qian Y-M, et al. Characterization of binding of leukotriene $\mathrm{C}_{4}$ by human multidrug resistance protein 1. Evidence of differential interactions with $\mathrm{NH}_{2}$ - and $\mathrm{COOH}$-proximal halves of the protein. J. Biol. Chem 2001;276:38636-38644. [PubMed: 11507101]

26. Austermuhle MI, Hall JA, Klug CS, Davidson AL. Maltose-binding protein is open in the catalytic transition state for ATP hydrolysis during maltose transport. J. Biol. Chem 2004;279:28243-28250. [PubMed: 15117946]

27. Hopfner K-P, Tainer JA. Rad50/SMC proteins and ABC transporters: unifying concepts from highresolution structures. Curr. Opin. Struct. Biol 2003;13:249-255. [PubMed: 12727520]

28. Csanády L. Rapid kinetic analysis of multichannel records by a simultaneous fit to all dwell-time histograms. Biophys. J 2000;78:785-799. [PubMed: 10653791] 
a
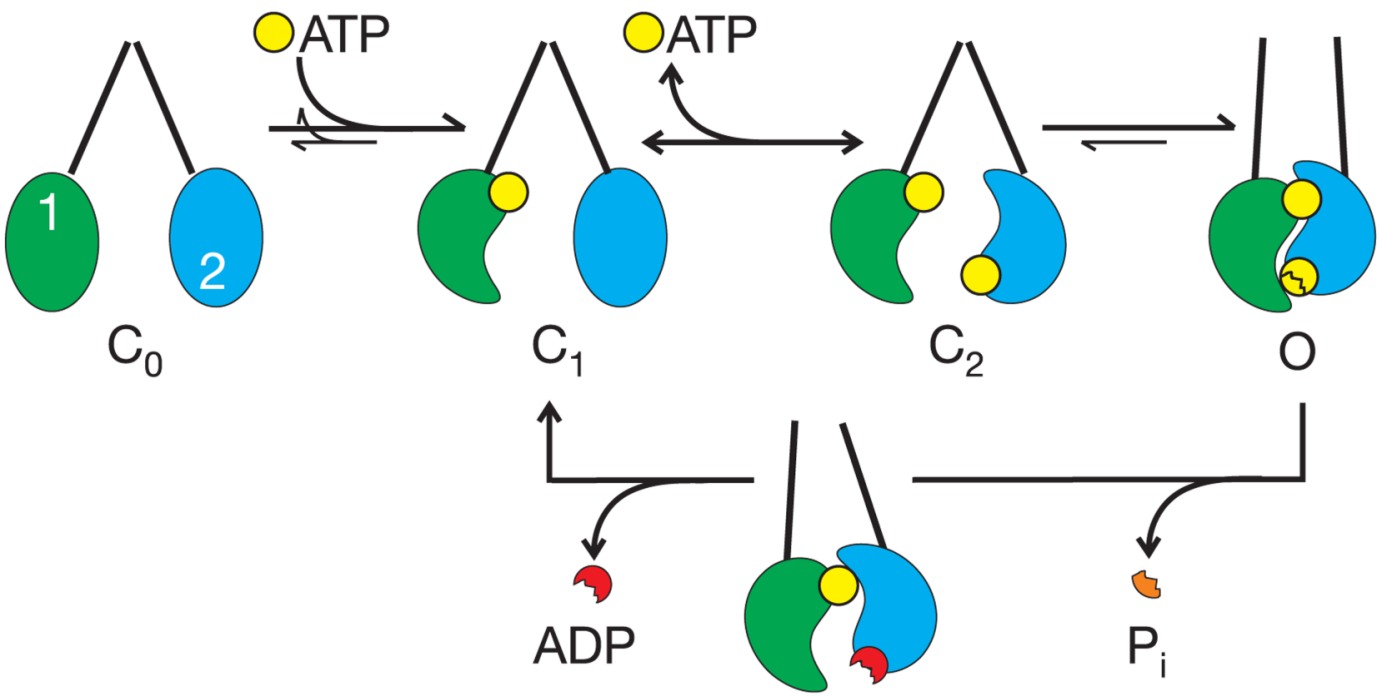

b

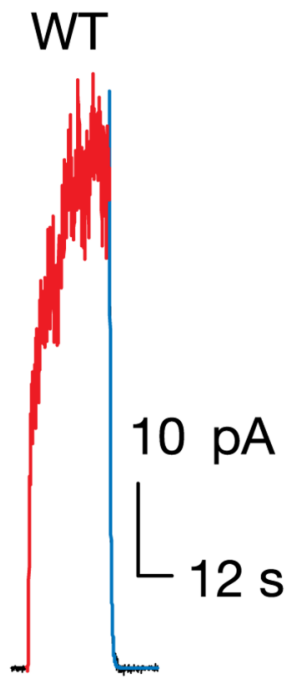

E1371Q

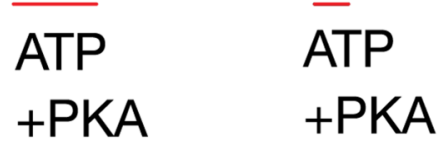

Figure 1.

Open CFTR channels correspond to dimerized NBDs. a, Diagram illustrating the proposed mechanism coupling the opening of the $\mathrm{Cl}^{-}$channel pore $\left(\mathrm{C}_{n}\right.$, closed states; $\mathrm{O}$, open $)$ in the transmembrane domains (converging, or semi-parallel, straight lines) to the hydrolysis cycle through the dimerization of NBDs (green, NBD1; blue, NBD2). The dynamic formation and disruption of a tight NBD dimer interface are represented by major changes in shape and position simply for clarity (see text). b, Mutating the 'Walker B' glutamate, Glu 1371, in NBD2 markedly increases the stability of the $\mathrm{Cl}^{-}$channel's open burst state. Records from patches containing hundreds of channels, activated by exposure to 5mM ATP and $300 \mathrm{nM}$ cAMP- 
dependent protein kinase (PKA, red). Time constants for current decay fit lines (blue): WT, $\tau=0.45 \mathrm{~s} ; \mathrm{E} 1371 \mathrm{Q}, \tau=476 \mathrm{~s}$. Note the fivefold expanded timescale for the WT record. 
a

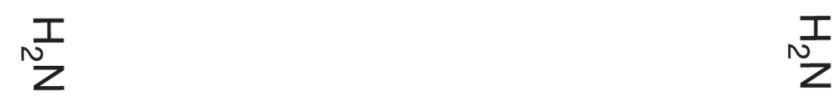

$\mathrm{R}$

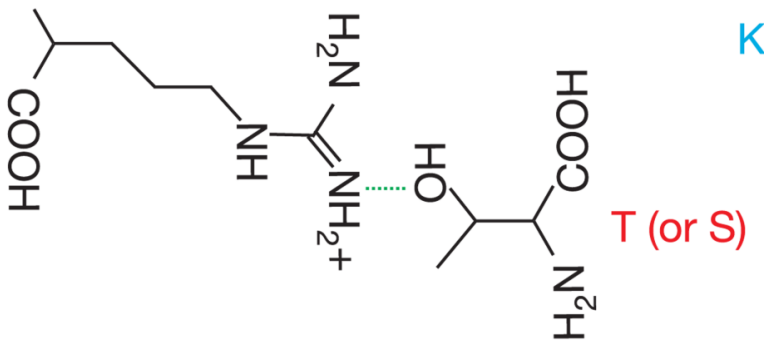

$\underset{Z}{I}$

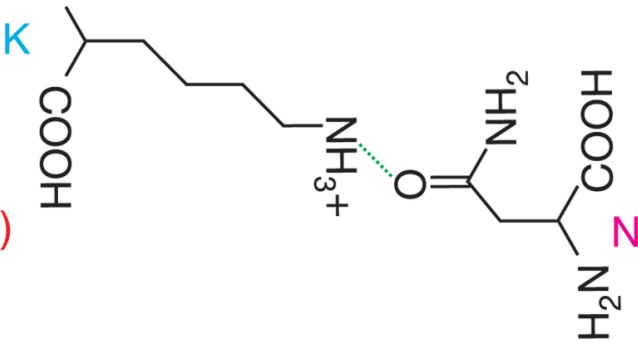

b

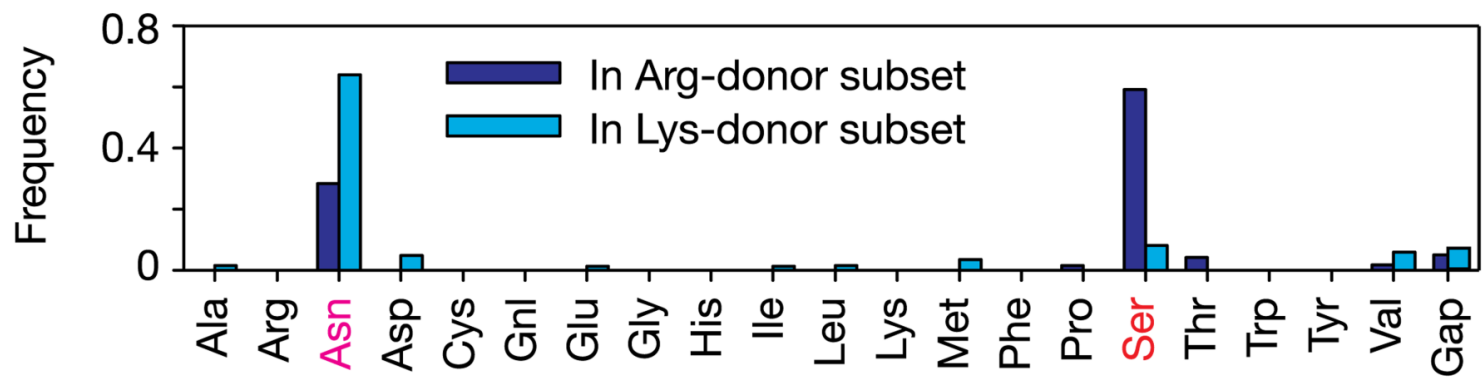

C

WT

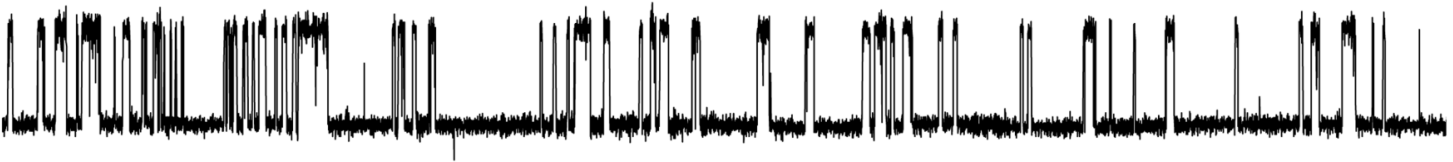

$\mathrm{R} 555 \mathrm{Q}$

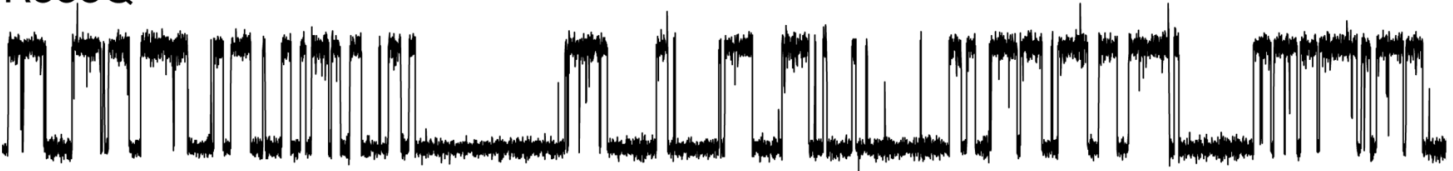

R555K

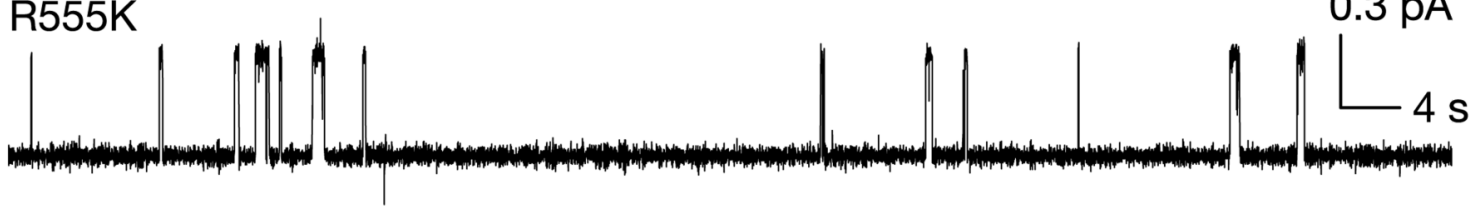

Figure 2.

Statistical coupling analysis and electrophysiological recordings position CFTR's Arg 555 in the composite NBD2 catalytic site. a, Representation of hydrogen-bonded arginine-threonine $(\mathrm{R}-\mathrm{T})$ and lysine-asparagine $(\mathrm{K}-\mathrm{N})$ pairs. In serine and threonine (the side chain present in CFTR) the acceptor oxygen atom is positioned at the same distance from the peptide backbone. b, Amino acid frequencies at the 'head' position, equivalent to Thr 1246, in alignment subsets having Arg (blue bars) or Lys (cyan bars) at the 'tail' position. The total alignment contained 10,194 sequences (http://www.sanger.ac.uk/cgi-bin/Pfam/getacc?PF00005). c, Mutations at Arg 555 in NBD1 'tail' affect mean open burst (R555Q) and closed interburst (R555K) dwell times. R555Q opened with rates comparable to WT (R555Q, $\tau_{\mathrm{ib}}=2.84 \pm 0.53 \mathrm{~s}(15)$ ) despite 
expected loss of the interfacial hydrogen bond, suggesting possible compensation by other factors, for example the removal of repulsive electrostatic forces. 


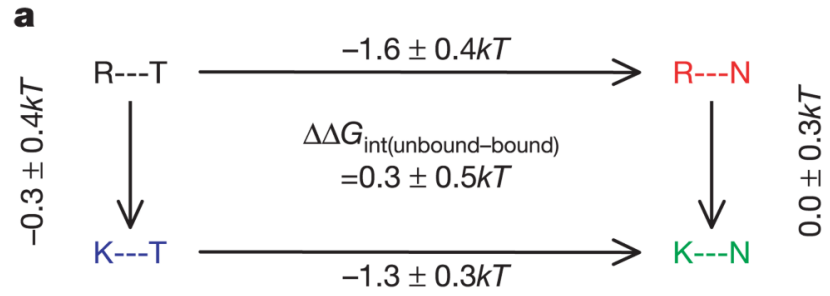

b
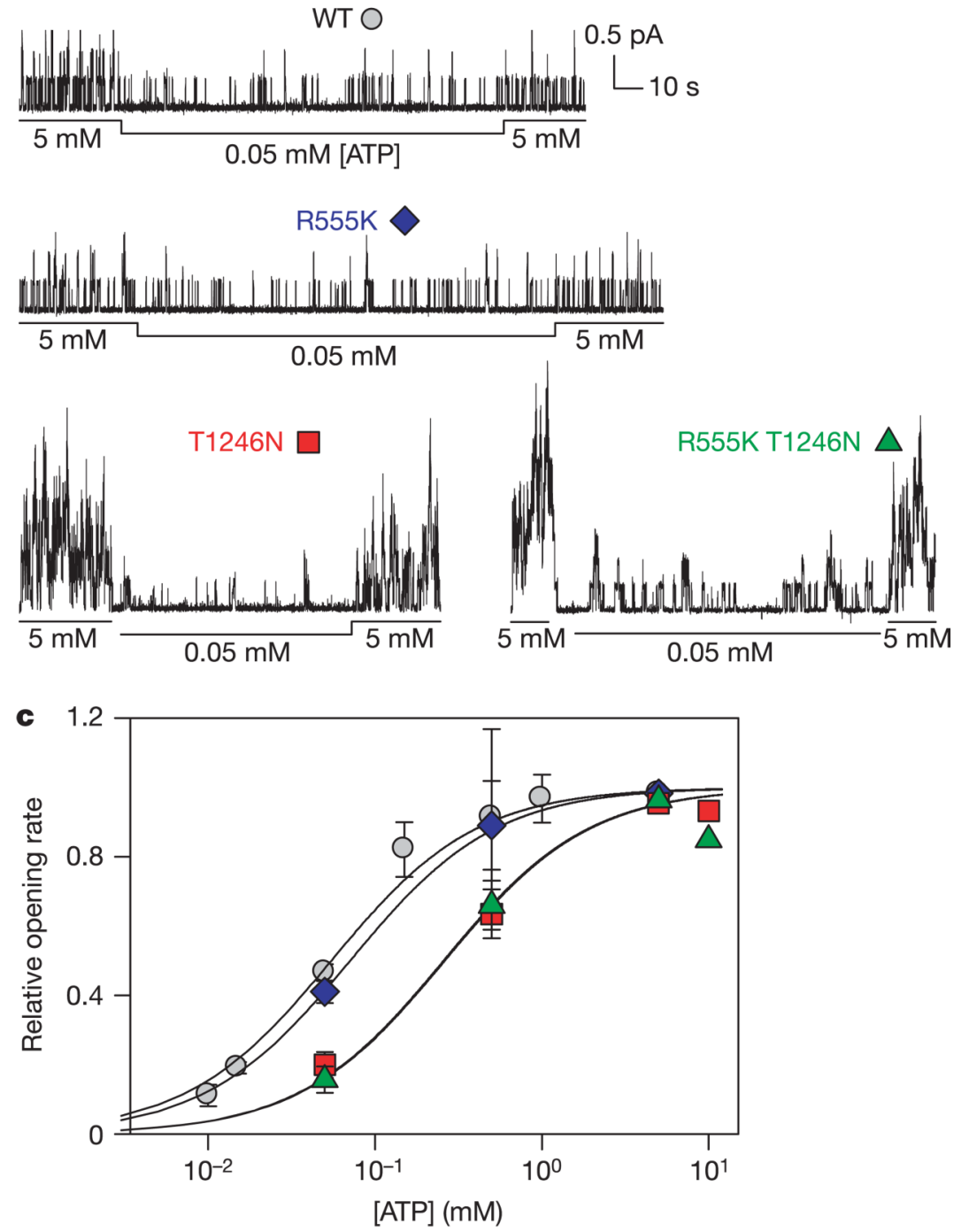

Figure 3.

Arg 555 and Thr 1246 are not energetically coupled in channel closed states. a, Thermodynamic mutant cycle (each corner defined by the side chains at position 555 and 1246) using changes in apparent dissociation constant $\left(K_{0.5}\right)$ as an estimate of the equilibrium constant for the reaction $\mathrm{C}_{2} \rightleftharpoons \mathrm{C}_{1}+\mathrm{ATP}$ (Fig. 1a). Values show changes (means \pm s.d.) in free energy difference driving this reaction under standard conditions, $\Delta G^{\circ}(\mathrm{C} 1+\mathrm{ATP})-\mathrm{C} 2=-k T \ln K_{0.5}(k$ is Boltzmann's constant, $T$ is absolute temperature). b, Representative records from WT, single mutants R555K and T1246N, and double mutant R555K T1246N. Exposure to the test [ATP] 
(here $50 \mu \mathrm{M}$ ) was bracketed by exposures to $5 \mathrm{mM}$ ATP. c, Mean relative ${ }^{14}$ opening rates ( \pm s.e.m., with $2 \leq n \leq 25$ ). 
a

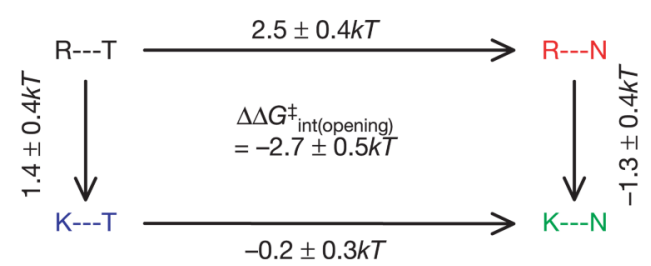

b

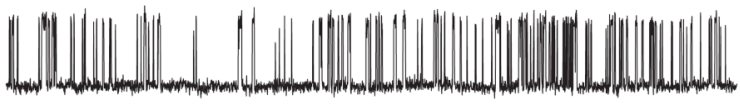

WT

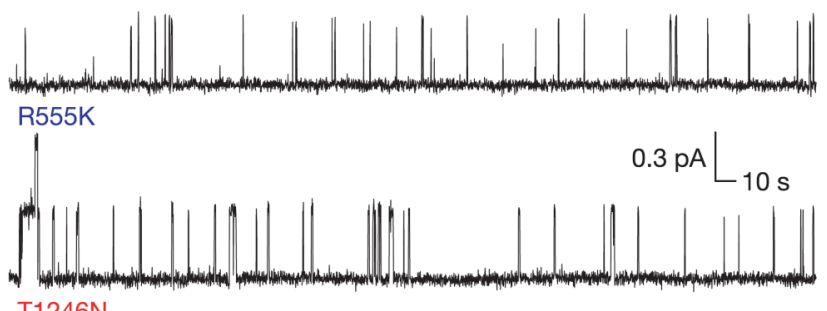

T1246N

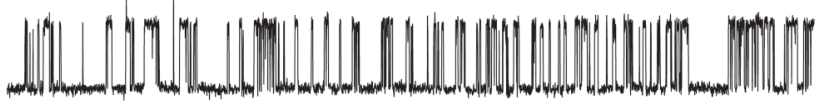

R555K T1246N

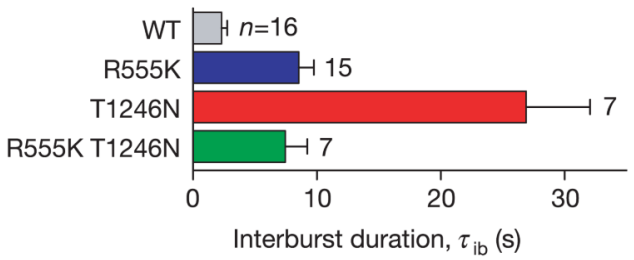

d

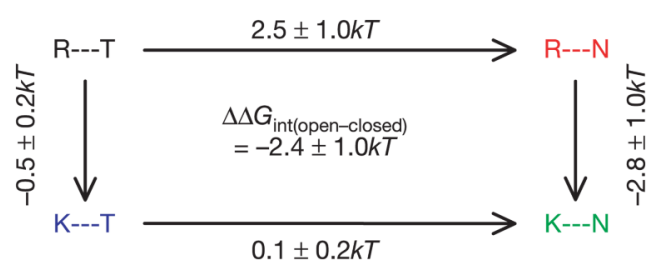

e

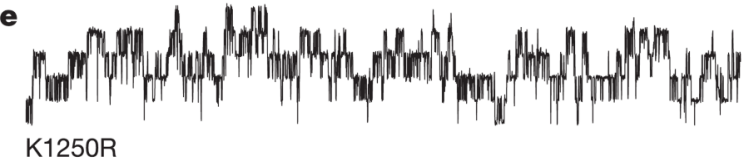

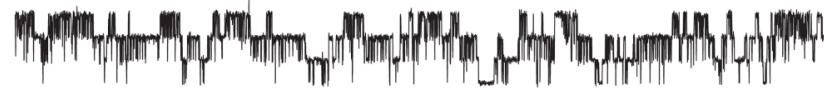

R555K K1250R
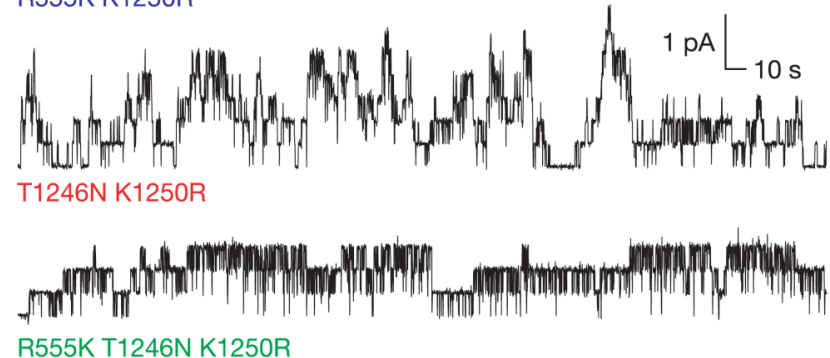

$\mathbf{f}$

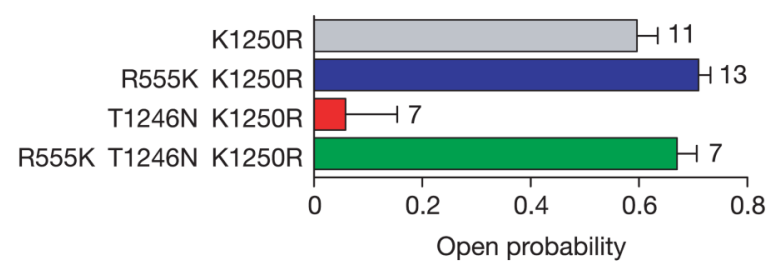

Figure 4.

Energetic coupling between Arg 555 and Thr 1246 accompanies channel opening. a,

Thermodynamic cycle showing changes (means \pm s.d.) in the activation energy barrier for opening $\left(\Delta \Delta G^{\ddagger}\right)$. b, Representative records. c, Mean closed interburst duration ( \pm s.e.m.). d, Thermodynamic cycle showing changes (means \pm s.d.) in stability of open state with respect to the closed state, $\Delta \Delta G$ (open-closed), calculated from $P_{\mathrm{o}}$. e, Representative records. Current levels of the triple mutant R555K T1246N K1250R did not change when [ATP] was increased to $10 \mathrm{mM}$, indicating that $5 \mathrm{mM}[\mathrm{ATP}]$ was saturating. $\mathbf{f}$, Mean $P_{\mathrm{o}}$ ( \pm s.e.m.). 